\title{
ARTICLE
}

\section{Sexual dysfunction: classification and assessment ${ }^{\dagger}$}

\author{
Dinesh Bhugra \& Gabriele Colombini
}

\begin{abstract}
Dinesh Bhugra is Professor
of Mental Health and Cultural Diversity at King's College London. $\mathrm{He}$ is also an honorary consultant at the Maudsley Hospital, where he runs the sexual and couples therapy clinic. Gabriele Colombini is training in psychiatry at the University of Pavia, Italy, and is an honorary researcher in the Health Service and Population Research Department at the Institute of Psychiatry, King's College London. Correspondence Professor Dinesh Bhugra, Health Service and Population Research Department, Institute of Psychiatry, King's College London, London SE5 8AF, UK. Email: dinesh.bhugra@kcl.ac.uk
\end{abstract}

†' the next issue of Advances, Vishal Bhavsar \& Dinesh Bhugra continue the discussion of sexual dysfunction in an article entitled 'Cultural factors and sexual dysfunction in clinical practice'

\begin{abstract}
SUMMARY
Sexual dysfunction is one of the most common psychiatric disorders, but it is often ignored in assessment. It can be primary or secondary (a result of psychiatric disorder or medication). Success rates in managing sexual dysfunction are relatively high, with good response to psychological and medical interventions. In ICD-10 and DSM-IV-TR, sexual dysfunctions are broadly classified on the basis of the stages of sexual activity, from arousal to orgasm. There are major similarities between ICD and DSM in diagnosis and classification of sexual dysfunction, but both systems raise challenges. These include definitions of what is 'normal' and how abnormality is defined. In this article, we describe the role of the two systems and possible amendments that might help researchers and clinicians. We also present key principles for the assessment and treatment of people who experience sexual dysfunction. We consider problems that need to be managed in engaging and in the therapeutic alliance.
\end{abstract}

\section{DECLARATION OF INTEREST}

None.

Psychosexual medicine and our understanding of sexual dysfunction have developed at a tremendous pace in the past half century. Although sexual dysfunction has very high rates of prevalence, for a number of reasons psychosexual disorders are often ignored by patients and clinicians alike. But this too is beginning to change, and we are seeing a lessening of the stigma related to help-seeking for sexual problems. However, secondary sexual dysfunction as a result of physical or psychiatric conditions carries with it both ignorance (because very often clinicians are not aware of it and do not enquire about it) and stigma (patients with mental illness are not expected to have sex or even talk about it). The field of sexual dysfunction and sexual therapy is one in which people are more likely to be highly motivated in seeking help and participating in treatment. Nevertheless, they and their partners often seek help elsewhere - from the internet or from self-help books. Later in this article, we will describe basic problems related to assessment of the type and degree of sexual dysfunction. First, we will discuss its diagnostic classification.

\section{Current classification}

Over the past 50 years or so, the taxonomy of sexual dysfunction in the ICD and DSM classifications has developed in parallel with research in the field. Generally, sexual problems are divided into three broad categories: sexual dysfunction, gender identity disorder and paraphilias. In this article we focus on sexual dysfunction.

In men, 'normal' stages of sexual function are arousal, erection, the sexual act and ejaculation, followed by a latency period. In women, these stages are arousal, the sexual act and orgasm. Dysfunction can occur at any of these stages. In addition, both men and women can experience pain during the sexual act. Both ICD-10 and DSM-IV-TR use these stages to classify sexual dysfunction (Table 1).

The context in which problems occur and present is important and clinicians must be aware of it, especially with regard to sexual orientation and relationships. There may be problems in the relationship, but not in the couple's sexual activity; equally, there may be sexual dysfunction, but not difficulties in the relationship (although, in our clinical experience, this is unlikely). Furthermore, the type of relationship (for example, between homosexual partners whether the relationship is open or closed) may influence the presentation of sexual dysfunction. Physical factors and age may also play a role. Relationships may need to be assessed with each partner separately and then together as a couple, to ensure that sexual dysfunction can be understood and treated in that context. Some of these underlying factors will influence the motivation and decision to seek help. They are not part of the classification but we will return to them later in this article, in our discussion of assessment.

\section{Challenges in classification}

As with other psychiatric conditions, there is a challenge in identifying the degree of dysfunction and deciding whether it is qualitatively different from normal or is described by professionals as 'abnormal' at the behest of society. Classification of psychiatric disorders brings with it specific issues related to abnormality and deviancy, how 
they are defined and who defines them. The distinction between categorical and dimensional aspects of disorder is critical in our understanding of psychiatric disorders, where objective tests are few and far between. In sexual dysfunction, an additional factor must be taken into account: as sex generally involves at least two people, the relationship between the couple plays a significant role in the genesis and perpetuation of sexual dysfunction. If other aspects of the relationship are fine and sexual dysfunction is the only problem, the focus has to be on managing the dysfunction. On the other hand, if the couple have other problems - whether these are individual or affect both - any diagnosis will need to take them into account.

In this section we will consider contextual factors that challenge traditional systems of classification.

\section{Abnormal v. normal}

One of the major challenges is how abnormality is defined and who defines it. For example, the degree of vaginismus can be measured objectively using a dilator of different sizes. But in the case of premature ejaculation, what is meant by premature? Does it refer to time, and is that in seconds or minutes? Does it mean that either partner has not reached full satisfaction? Similarly, when considering or assessing low sexual desire, how low is low? Of the two classificatory systems, DSM-IV-TR does include as a criterion that lack of desire must cause marked distress or interpersonal difficulty. However, there is no method to measure what is a marked aspect of distress or interpersonal difficulty. If the patient's problem occurs with one particular sexual partner but not with others, it is particularly challenging for the diagnostician to assess the degree of distress or interpersonal difficulty. The cause and effect of distress and dysfunction need to be ascertained and understood in context. Dissatisfaction with penile size can cause distress, but the nature of the distress may be disproportionate. How is the size of the penis determined to be 'normal'?

\section{Categories v. dimensions}

Both ICD-10 and DSM-IV use an essentially categorical (dichotomous) classification of disorders. However, the plans for DSM-5 and ICD-11 suggest a move away from categories to dimensions. It will be helpful to determine how this shift will be effected in cases where more than one category is present. Although it may be possible to mark dimensions or degrees of disorder on a scale, categories may overlap. For example, low sexual desire can lead to erectile difficulty and vice versa. Also, dimensions may well be
TABLE 1 Classification of sexual dysfunctions

\begin{tabular}{|c|c|}
\hline DSM-IV-TR & ICD-10 \\
\hline & $\begin{array}{l}\text { Block F52: sexual dysfunction not caused by } \\
\text { organic disorder }\end{array}$ \\
\hline \multicolumn{2}{|l|}{ Disorders of desire } \\
\hline 302.71 Hypoactive sexual desire disorder & F52.0 Lack/loss of sexual desire \\
\hline \multirow[t]{2}{*}{ 302.79 Sexual aversion disorder } & $\begin{array}{l}\text { F52.1 Sexual aversion/lack of sexual } \\
\text { enjoyment }\end{array}$ \\
\hline & F52.7 Excessive sexual drive \\
\hline \multicolumn{2}{|l|}{ Disorders of arousal (vaginal dryness/impotence) } \\
\hline 302.72 Female sexual arousal disorder & F52.2 Failure of genital response \\
\hline \multicolumn{2}{|l|}{ 302.72 Male erectile disorder } \\
\hline \multicolumn{2}{|c|}{ Disorders of orgasm (delay, absence, premature ejaculation) } \\
\hline 302.73 Female orgasmic disorder & F52.3 Orgasmic dysfunction \\
\hline \multicolumn{2}{|l|}{ 302.74 Male orgasmic disorder } \\
\hline 302.75 Premature ejaculation & F52.4 Premature ejaculation \\
\hline \multicolumn{2}{|l|}{ Disorders of sexual pain } \\
\hline $\begin{array}{l}\text { 302.76 Dyspareunia (not due to general } \\
\text { medical condition) }\end{array}$ & F52.6 Non-organic dyspareunia \\
\hline $\begin{array}{l}302.51 \text { Vaginismus (not due to general } \\
\text { medical condition) }\end{array}$ & F52.5 Non-organic vaginismus \\
\hline \multicolumn{2}{|l|}{ Other } \\
\hline \multicolumn{2}{|l|}{$\begin{array}{l}625.8 \text { (Female), } 608.89 \text { (Male) sexual } \\
\text { dysfunction due to general medical condition }\end{array}$} \\
\hline \multicolumn{2}{|l|}{ 291.89 Substance-induced dysfunction } \\
\hline \multirow[t]{2}{*}{$\begin{array}{l}\text { 302.70 Sexual dysfunction not otherwise } \\
\text { specified (NOS) }\end{array}$} & $\begin{array}{l}\text { F52.8 Other sexual dysfunction, not caused } \\
\text { by organic disorder or disease }\end{array}$ \\
\hline & $\begin{array}{l}\text { F52.9 Unspecified sexual dysfunction, not } \\
\text { caused by organic disorder or disease }\end{array}$ \\
\hline
\end{tabular}

Sources: DSM-IV-TR: American Psychiatric Association 2000; ICD-10, World Health Organization 1992.

linear, but psychiatric conditions often affect more than one dimension, especially in the field of sexual dysfunction. Expectations of performance are strongly influenced by social mores and social presuppositions. The distress experienced from sexual dysfunction may be a result of social disapproval. In making sense of sexual dysfunction, dimensions and categories equally require a sensible and pragmatic understanding of social norms and cultural contexts.

\section{Organic v. functional}

In psychiatric conditions, a distinction is often made between the organic and the functional. However, this distinction is not always clear and, even if it is, measurements might not differentiate between slight neural damage and poor psychological functioning. Biological psychiatrists see abnormal neural structures and neural damage as the key to making a diagnosis; psychological symptoms may be secondary in the diagnostic process. However, neural damage is not always evident, or even present, in psychological illness. 
A difference in their biological substrate can make individuals vulnerable to certain conditions or behaviour. In the context of sexual dysfunction, this substrate may be not in the brain but elsewhere - in the peripheral nervous system or the genitalia (in the nerves or vascular supply). The distinction between organic and functional becomes intriguing in the context of diagnosing and clarifying sexual dysfunction. The example of erectile dysfunction may make this clear. It may have an organic cause (such as diabetes, peripheral vascular disease or venous leaks), but labelling it functional indicates that it is caused by anxiety, whether that anxiety is primary, and thus causal, or secondary to the underlying organic disorder.

\section{ICD-10 and DSM-IV-TR}

The DSM and ICD diagnostic and classificatory systems deal with organic sexual dysfunction in different ways. DSM-IV-TR has seven diagnostic categories under the broad rubric of sexual dysfunction due to a general medical condition (Table 2). It also has a category for substanceinduced sexual dysfunction, whereas ICD-10 has no such category. In ICD-10, dysfunctions of organic origin are meant to be classified elsewhere in the manual.

Although the ICD and DSM systems are entirely categorical, in both cases, many of these categories are based on dimensions without any clear demarcation between normal and abnormal. For example, both systems contain a diagnostic category in which abnormally low sexual desire is the cardinal symptom (F52.0 in ICD-10, noted as lack or loss of sexual desire; and 302.71 in DSM-IV-TR, noted as hypoactive sexual desire disorder), but neither attempts to give a benchmark of 'normal' against which to assess it. DSM-IVTR has five axes, addressing: clinical disorder; personality disorder; general medical conditions; psychosocial and environmental factors contributing to disorder; and global functioning.

\section{TABLE 2 DSM-IV-TR classification of sexual dysfunc-} tion due to a general medical condition

\begin{tabular}{|ll|}
\hline \multicolumn{2}{|c|}{} \\
\hline 625.8 & Female hypoactive sexual desire disorder due to... \\
\hline 608.89 & Male hypoactive sexual desire disorder due to... \\
\hline 607.84 & Male erectile disorder due to... \\
\hline 625.0 & Female dyspareunia due to... \\
\hline 608.89 & Male dyspareunia due to... \\
\hline 625.8 & Other female sexual dysfunction due to... \\
\hline 608.89 & Other male sexual dysfunction due to... \\
\hline
\end{tabular}

Source: American Psychiatric Association 2000.
ICD-10 alludes to a multi-axial approach but does not take this further; whether this will change in ICD-11 remains to be seen.

Unlike DSM-IV-TR, ICD-10 has no specific category for substance-induced sexual dysfunction (Table 1). However, DSM-IV-TR does not have excessive sexual drive as a category. ICD-10 groups disorders of arousal and orgasm across sexes in the same category.

In its diagnostic categories, DSM-IV-TR provides more detailed descriptions of symptoms than ICD-10. It also makes a distinction between duration (lifelong or acquired), situation (generalised to all or some partners), and the relative contribution of organic and psychological factors.

\section{Clinical reality}

Placing disorders of sexual dysfunction in neat categories is often difficult in clinical practice. Even though these disorders have a physical or psychological basis, often they sit uncomfortably in the realm of clinical psychiatry. In many clinical settings, urologists or gynaecologists treat some of these problems. Sexually transmitted disease (STD) clinics also provide some interventions. The challenge for psychiatry therefore is to combine the physical and psychological factors and integrate them effectively in both understanding aetiology and developing management plans.

\section{Comorbidity}

Although clear data are not available, sexual dysfunction does tend to have associated physical and psychiatric problems. Often, dysfunction itself is secondary to psychiatric disorder. Both systems allow comorbidity in diagnosis and co-occurrence of various sexual dysfunctions, which means that several may be diagnosed in an individual, although this is not always easy to do.

In order to deliver appropriate therapeutic intervention and for improved outcomes, careful consideration must be given to diagnosing and classifying sexual dysfunction, especially in the presence of comorbid conditions.

\section{Sexual maturation and development}

ICD-10 has a category not included in DSM-IV: 'F66 Psychological and behavioural disorders associated with sexual development and orientation'. Distress about sexual orientation can take several forms. In ICD-10, F66.1 deals with ego-dystonic sexual dysfunction, the core feature of which is that sexual orientation in itself is not in doubt but the individual wishes that it were different. This category is implicitly for those who find themselves homosexual but wish they were 
not. Further, F66.2 deals with sexual relationship disorder, where abnormality of gender identity or sexual preference is causing difficulties in forming or maintaining sexual relationships, while F66.3 - sexual maturation disorder - is reserved for those (particularly adolescents) who are uncertain whether they are homosexual, heterosexual or bisexual and are suffering distress as a result. This category also includes those who find that their sexual orientation is changing after a long period of stability, leading to uncertainty and distress. These categories appear in DSM-IV as 'sexual disorder not otherwise specified'. This discrepancy between the two systems raises the question about social context and whether these categories have any use in everyday clinical practice.

\section{Sexual dysfunction in clinical practice}

\section{Types of sexual dysfunction}

As outlined above, sexual dysfunction is classified generally in terms of disorders of desire, arousal and intercourse (specifically pain during the sexual act), and phobia (Table 3). DSM-IV-TR divides these into lifelong (primary) or acquired (secondary). Sexual dysfunction may also be generalised (occurring across all sexual situations and with all partners) or situational (only with some partners in some situations). These distinctions can be arbitrary: for example, how many partners and how many times? However, they are helpful as they may enable the clinician to understand psychological and social aspects of the dysfunction. It is worth remembering that couples may have more than one dysfunction between the two of them and dysfunctions may not be entirely discrete.

Sexual function involves fantasy and behaviour. The diagnostic concept of sexual dysfunction encompasses various ways in which an individual is unable to participate in a sexual relationship as they would wish. For example, lowering or absence of desire or a discrepancy in desire between sexual partners at any stage can produce dysfunction. Sexual dysfunction needs to be differentiated from sexual deviation or paraphilias (recurrent intense sexual urges and experiences that can focus on non-human objects, on the self and on nonconsenting partners) and general dissatisfaction. Self-reported dissatisfaction also needs to be noted in any assessment along with distress reported by the partners. It becomes especially relevant if individuals also report dissatisfaction with sexual performance or with body parts. Dissatisfaction may not lead to dysfunction but the reverse is certainly true. Additional aetiological precipitants may also play a role.
TABLE 3 General classification of sexual dysfunction

\begin{tabular}{|lll|}
\hline Phase & Male & Female \\
\hline Desire/drive/interest & $\begin{array}{l}\text { Low interest } \\
\text { Excessive interest }\end{array}$ & Low interest \\
& Excessive interest \\
\hline Arousal/excitement & Erectile dysfunction & Impaired arousal \\
& Premature ejaculation & Anorgasmia \\
& Retarded ejaculation & \\
\hline Sexual pain/other & Dyspareunia & Dyspareunia \\
& Sexual phobias & Vaginismus \\
& & Sexual phobias \\
\hline
\end{tabular}

\section{Prevalence of sexual dysfunction}

A major problem in getting accurate measures of the prevalence of sexual dysfunction is sample selection. Prevalence rates will vary depending on where the sample is collected. Thus, it is inevitable that rates will be high among those attending medical, urological and gynaecological clinics. Population surveys give better estimates, but again they depend on sample selection and how the questions are asked.

Masters \& Johnson (1970) estimated that $50 \%$ of all couples in the USA have a sexual dysfunction. In a study of 100 White, happily married and educated couples in the USA, $40 \%$ of the men reported ejaculatory or orgasmic dysfunction (Frank 1978). The same rate was reported by the men among 58 married Swedish couples (Moreira 1979). Interestingly, sexual dysfunction was not related to sexual satisfaction. Robins et al (1984) estimated that $24 \%$ of the US population at some point in their life would have a sexual dysfunction. Laumann et al (1999) reported from a national probability sample of 1410 men and 1749 women (aged 18-59) that $43 \%$ of women had sexual dysfunction, compared with $31 \%$ of men. Poor physical and emotional health and ageing were associated with higher rates. Not surprisingly, they found that sexual dysfunction was strongly associated with negative experiences in sexual relationships and with overall well-being. A subsequent global study by Laumann et al (2005) showed prevalence of sexual dysfunction in women at $38 \%$ and in men at 29\%. Moreira et al (2005) and Laumann et al (2005) reported on a total sample of 27500 participants (aged between 40 and 80) from 29 countries. Almost half had experienced at least one sexual problem, but less than 1 in 5 had sought medical help. Among 6700 participants (aged 4080) in 9 Asian countries, $20 \%$ of men and 30\% of women reported sexual problems (Nicolosi 2005). The sampling technique varied across countries and the response rate was around 33\%. Age was a significant variable in a number of conditions. The sample analysis included only those who were 
sexually active. Thus, those whose dysfunction was serious enough to stop them having sex altogether were excluded completely. These studies highlight high levels of sexual dysfunction irrespective of culture, although cross-cultural prevalence data are limited.

Cultures influence beliefs and customs and may precipitate or perpetuate symptoms. It is possible that sexual dysfunction is more prevalent in what Bullough (1976) described as 'sex-positive' cultures (where the sexual act is seen as fun and not necessarily procreative).

In a study of erectile dysfunction in communitybased populations in Brazil, Italy, Japan and Malaysia, the age-adjusted prevalence varied from $15 \%$ in Brazil to more than twice that $(34 \%)$ in Japan (Nicolosi 2003). More than half of the men in samples from Morocco (54\%; Berrada 2003) and Nairobi (57\%; Sebit 1995) reported erectile dysfunction, and Ghanain women reported high levels of vulvar pain (Adanu 2005). In China, $28 \%$ of men and $46 \%$ of women reported at least one sexual dysfunction Parish 2002. The role of culture on the genesis and maintenance of sexual dysfunction will be discussed at some length in a forthcoming article in this journal (Bhavsar 2013).

\section{Sexual minority groups}

Prevalence rates for sexual dysfunction among groups such as lesbians, gay men and transgender individuals seem to be similar to those of the general population, although the research data are sparse. One study of 200 gay men attending a seminar noted that $97.5 \%$ reported sexual dysfunction over their lifetime and $52.5 \%$ had current concerns regarding sexual dysfunction (Rosser 1997). Bisexual individuals may have additional difficulties in that sexual dysfunction may occur with partners of one gender but not the other. Studies have indicated that sexual minority groups may have high rates of alcohol (Bergmark 1999) or ecstasy use (Klitzman 2002), thereby contributing to sexual dysfunction. Harmful substance use may be socially reinforced and regarded as normal by patients, so therapists need to be aware of norms and social expectations. An increase in unprotected sex and resulting higher rates of sexually transmitted diseases will add another dimension to help-seeking and prevalence of sexual dysfunction. Perceived or real homophobia and stages of coming out will also play a role.

\section{Older adults}

Older individuals, along with their sexuality and sexual needs, are often ignored by clinical services.
Older people may think of themselves as being too old for sex, possibly as a result of society's attitudes, and may think that sexual dysfunction is a result of ageing, whereas in reality it may be due to physical or psychiatric conditions.

\section{The clinical assessment}

The purpose of assessment for sexual dysfunction is summarised in Box 1. The assessment may be part of a medical assessment by a physician or surgeon or a psychological assessment in a special clinic dealing with sexual dysfunction. Two specific issues about confidentiality in this context need to be remembered. First, the interview should be conducted in a private space where it cannot be overheard. Second, in the assessment of couples individually, some ground rules need to be established. If one partner acknowledges that they are having sex elsewhere and therefore their libido is low or they are not able to perform with their partner, then the therapist is stuck with the information and may also be seen as colluding with that partner. It is best to make it clear to both partners that they must not tell the therapist anything that they would not wish to share with their partner. If something is inadvertently revealed, the therapist should encourage the individual to share it with the partner, either privately or during a session.

Most specialist clinics will have their own policies governing assessment interviews. Some send out screening questionnaires before the first appointment to ensure that the right therapist is available and a degree of matching between therapist expertise and patient needs is possible. Some patients will be referred simply for 'education' because they have sexual difficulties but not fullfledged dysfunction.

In sexual dysfunction perhaps more than in other psychiatric disorders, the interaction between biological, psychological and social (including cultural and interpersonal) factors is important in placing the patient in the right context and in planning any interventions.

Biological factors to be considered include physical illness, endocrinal abnormalities,

B0X 1 Aims of clinical assessment

- To define the dysfunction

- To assess whether it is organic or non-organic

- To assess immediate causes

- To assess resources and motivation

- To decide on correct management and prognosis 
diabetes, hypertension, cardiovascular, gynaecological and urological disorders, and iatrogenic effects of medication.

Psychological factors include stress, a poor relationship, clinical depression, anxiety, schizophrenia, history of sexual abuse, low self-esteem and other comorbid psychiatric disorders.

Social factors include interpersonal problems such a poor relationship, sexual inhibitions, affairs and fidelity-related matters, religious and sexual conflict, differences in cultural expectations and values, and child-bearing.

In any assessment, a broad exploration of factors in the three domains, with a gradual focus on understanding what may be the predominant features, will help. Reasons for attending the clinic and seeking help now, and the motivation for change, need to be explored. Both partners may contribute to this, either together or singly. The detailed interview should then explore the degree of dysfunction and the contribution of various aetiological factors, which can then be investigated and a degree of understanding about what is important can be obtained. Indications for physical examination are listed in Box 2.

The assessment interview may be started with both partners together, followed by individual interviews, and finally joint information-sharing, assessment and treatment advice. The aims of the assessment are to develop a clear picture of the problem, identify suitability for treatment and offer the right intervention. A poor relationship between the patient and their partner, poor motivation, severe psychiatric disorders, history of sexual assault, alcoholism and severe untreated physical illness are among the factors that are likely to result in poor engagement. Therapists also need to be aware of their own limitations (Box 3).

BOX 2 Indications for physical examination

- Recent history of physical ill health, presence of physical symptoms apart from sexual dysfunction

- Pain or discomfort during sexual activity

- Recent onset of loss of desire without any apparent cause

- Inability to produce a normal erection while awake (under any circumstances)

- Male aged over 50

- Female with sexual problems peri- or post-menopause

- History of marked menstrual irregularity and infertility

- History of abnormal puberty or endocrine disorder
BOX 3 Practical issues in dealing with sexual dysfunction

- Know your limitations - decide on the extent to which you can cope and get involved

- Are you being asked to conduct simply a brief assessment to be followed by a referral elsewhere?

- Refer if you feel that the patient's interests will be better served elsewhere

- If you are uncomfortable with a patient's fantasies, attitudes or behaviour, refer them on, for example to a counsellor, sex therapist, another psychiatrist, surgeon, gynaecologist, urologist or specialist clinic for erectile dysfunction

Box 4 outlines the areas to be covered during history-taking. The assessment needs a clear structure, although different components can be moved around. The therapist must be comfortable with the situation - the questions they ask and the words they use. Colloquial terms and slang should be avoided if possible. Questions should be open-ended, exploratory and non-critical. Research-related questioning will carry a different emphasis. Questionnaires and other assessment tools may be appropriate (see below). Box 5 lists laboratory tests that might may be useful. More specific investigations include Doppler sonography, phalloarteriography, cavernosometry, penile plethysmography and nocturnal penile tumescence testing.

\section{BOX 4 Broad headings for history-taking}

1 Sociodemographic factors: age, sexual orientation marital status, sexual activity

2 Presenting complaints: Why here? Why now? Past interventions, if any. Precise nature of the problem

3 History of sexual problem

4 Nature of general relationship with partner

5 Psychiatric history, including alcohol and substance misuse

6 Medical history, including smoking

7 Contraceptive history

8 Menstrual history

9 Sexual history - developmental, masturbation, schooling

Sexual fantasy

Sexual behaviour

10 Attitudes to the problem - religious beliefs, sexual knowledge

11 Attitudes to the intervention

12 Formulation 


MCO answers
$1 \mathrm{c} \quad 2 \mathrm{~b} \quad 3 \mathrm{~b} \quad 4 \mathrm{a} \quad 5 \mathrm{e}$

BOX 5 Suggested laboratory investigations

\section{Males}

- Blood count and erythrocyte sedimentation rate (ESR)

- Serum testosterone: total and free

- Sex hormone binding globulin (SHBG)

- Thyroid function test

- Liver function tests

- Renal function tests

- Lipid profiles

- Blood glucose levels

Females

- Blood count and ESR

- Thyroid function test

- Liver function tests

- Renal function tests

- Serum oestradiol

- Follicle stimulating hormone levels

- Prolactin levels

- Luteinising hormone levels

- Blood glucose levels

\section{Assessment tools}

Many assessment tools have been in use for a number of years and some are better known than others. Those listed in Table 4 are illustrative and should not be seen as recommendations or as the only tools available. Information on the validity and psychometric properties of some of these questionnaires is provided by Derogatis \& Balon (2009). Working across cultures may

\section{TABLE 4 A selection of assessment instruments}

\begin{tabular}{|lll|}
\hline Condition & Instrument & Gender \\
\hline Sexual desire & Arizona Sexual Experiences Scale (McGahuey 2000) & Both \\
& $\begin{array}{l}\text { Changes in Sexual Functioning Ouestionnaire (Clayton 1997) } \\
\text { Female Sexual Function Index (Rosen 2000) }\end{array}$ & Both \\
& Derogatis Interview for Sexual Functioning (Derogatis 1997) & Female \\
\hline Sexual arousal & Profile of Female Sexual Function (Derogatis 2004; McHorney 2004) & Female \\
& Sexual Function Questionnaire (Ouirk 2002) & Female \\
\hline Orgasm & Index of Premature Ejaculation (Althof 2006) & Male \\
& International Index of Erectile Function (Rosen 1997) & Male \\
\hline Sexual distress & Female Sexual Distress Scale (Derogatis 2002, 2008) & Female \\
\hline Overall ratings & Golombok-Rust Inventory of Sexual Satisfaction (Golombok 1985) & Both \\
& Golombok-Rust Inventory of Marital State (Rust 1989) & Both \\
& Sexual Interest and Desire Inventory - Female (Clayton 2006) & Female \\
& Short Personal Experiences Questionnaire (Dennerstein 2001) & Female \\
\hline
\end{tabular}

Adapted from Derogatis 2009. bring different factors and nuances into play, and validation for different populations may be required.

\section{Conclusions}

There are many similarities in approach and content across the DSM and ICD classificatory and diagnostic systems, although the axial approach in the DSM carries with it certain advantages that are useful in classifying sexual dysfunction. Nevertheless, the problems of defining normality, deviancy, type of dysfunction and dimensions in relation to psychiatric illnesses are reflected in classifying sexual dysfunction. An additional problem is that, more often than not, sexual dysfunction affects relationships and at least two people are involved. Researchers may find the categories in the current DSM and ICD helpful, but overlap and comorbidity may make it difficult to use them in routine clinical practice.

A thorough assessment and formulation are critical to ensuring that sexual functioning and dysfunction are understood in the context of the patient's personality, sexual relationships and sociocultural context. A comfortable atmosphere and total privacy for interviews and for physical examination, if necessary, are essential. Developmental history, past sexual abuse or assault, and sexual orientation all need to be explored gently and thoroughly. Thorough assessment will lead to optimal treatment. The challenge for the clinician is to remain professional and unshockable, no matter what is presented by the patient.

\section{References and further reading}

Adanu RM, Haefner HK, Reed BD, et al (2005) Vulvar pain in women attending a general medical clinic in Accra, Ghana. Journal of Reproductive Medicine 50: 130-4.

American Psychiatric Association (2000) Diagnostic and Statistical Manual of Mental Disorders (4th edn, Text Revised) (DSM-IV-TR). APA.

Althof S, Rosen R, Symonds T, et al (2006) Development and validation of a new questionnaire to assess sexual satisfaction, control and distress associated with premature ejaculation. Journal of Sexual Medicine 3: 465-75.

*Balon R (ed) (2008) Sexual Dysfunction. Karger.

*Balon R, Segraves RT (eds) (2009) Clinical Manual of Sexual Disorders. American Psychiatric Publishing.

*Bancroft J (2009) Human Sexuality and its Problems. Churchill Livingstone.

Bergmark KH (1999) Drinking in the Swedish gay and lesbian community. Drug and Alcohol Dependence 56: 133-43.

Berrada S, Kadri N, Mechakra-Tahiri S, et al (2003) Prevalence of erectile dysfunction and its correlates: a population based study in Morocco. International Journal of Impotence Research 15 (suppl 1): 53-7.

Bhavsar VB, Bhugra D (2013) Cultural factors and sexual dysfunction in clinical practice. Advances in Psychiatric Treatment in press.

Bullough V (1976) Sexual Variance in Society and History. University of Chicago Press. 
Clayton AH, McGarvey E, Claver G, et al (1997) Comparison of sexual functioning in clinical and non-clinical populations using the Changes in Sexual Functioning Questionnaire. Psychopharmacology Bulletin 33: 747-53.

Clayton AH, Segraves RT, Leiblum S, et al (2006) Reliability and validity of the Sexual Interest and Desire Inventory-Female (SIDI-F): a scale designed to measure severity of female hypoactive sexual desire disorder. Journal of Sex and Marital Therapy 32: 115-35.

Dennerstein L, Lehrer P, Dudley E (2001) Short scale to measure female sexuality: adapted from McCoy Female Sexuality Questionnaire. Journal of Sex and Marital Therapy 23: 291-304.

Derogatis LR (1997) The Derogatis Interview for Sexual Functioning (DISF/DISF-SR): an introductory report. Journal of Sex and Marital Therapy 23: 291-304.

Derogatis LR, Rosen RC, Leiblum S, et al (2002) The Female Sexual Distress Scale: initial validation of a standardised scale for the assessment of sexually related personal distress in women. Journal of Sex and Marital Therapy 28: 317-30.

Derogatis LR, Rust J, Golombok S, et al (2004) Validation of the Profile of Female Sexual Function (PFSF) in surgically and naturally menopausal women. Journal of Sex and Marital Therapy 30: 25-36.

Derogatis LR, Clayton A, Lewis-Di Agostino D, et al (2008) Validation of the Female Sexual Distress Scale - Revised (FSDS-R) for assessing distress in women with hypoactive sexual desire disorder. Journal of Sex and Marital Therapy 5: 357-64.

Derogatis LR, Balon R, (2009) Clinical evaluation of sexual dysfunctions. In Clinical Manual of Sexual Disorders (eds R Balon, RT Segraves): 25-58. American Psychiatric Publishing

Frank E, Anderson C, Rubinstein D (1978) Frequency of sexual dysfunction in 'normal' couples. New England Journal of Medicine 299: 111-5.

Golombok S, Rust J (1985) The Golombok-Rust Inventory of Sexual Satisfaction. British Journal of Clinical Psychology 24: 63-4.

Klitzman R, Greenberg J, Pollack M, et al (2002) MDMA ('ecstasy') use and its association with high risk behaviors, mental health and other factors among gay/bisexual men in New York City. Drug and Alcohol Dependence 661: 115-25.

Laumann EO, Paik A, Rosen RC (1999) Sexual dysfunction in the United States: prevalence and predictors. JAMA 281: 537-44.

Laumann EO, Nicolosi A, Glasser DB, et al (2005) Sexual problems among men and women aged 40-80 years. International Journal of Impotence Research 17: 39-57.

*LeVay S, Baldwin J (2009) Human Sexuality. Sinaeur Associates

Masters W, Johnson V (1970) Human Sexual Inadequacy. Little Brown.

McGahuey C, Gelenberg A, Laukes CA, et al (2000) The Arizona Sexual Experience Scale (ASEX) reliability and validity. Journal of Sex and Marital Therapy 26: 25-40.
McHorney CA, Rust J, Golombok S, et al (2004) Profile of Female Sexual Function: a patient-based, international, psychometric instrument for the assessment of hypoactive sexual desire in oophorectomized women. Menopause 11: 474-83.

Moreira JR, Nettelbladt P, Uddenberg N (1979) Sexual dysfunction and sexual satisfaction in 58 married Swedish men. Journal of Psychosomatic Research 23: 141-7.

Moreira E, Brock G, Glasser D, et al (2005) Help seeking behaviour for sexual problems: the Global Study of Sexual Attitudes and Behaviours. International Journal of Clinical Practice 59: 6-16.

Nicolosi A, Moreira E, Shirai M, et al (2003) Epidemiology of erectile dysfunction in four countries: cross-national study of the prevalence and correlates of erectile dysfunction. Urology 61: 201-6.

Nicolosi A, Glasser D, Kim S, et al (2005) Sexual behaviour and dysfunction and help-seeking patterns in adults aged $40-80$ years in the urban population of Asian countries. British Journal of Urology International 95: 605-14.

Parish WL, Laumann E0, Pan S, et al (2007) Sexual dysfunctions in urban China: a population-based national survey of men and women. Journal of Sexual Medicine 4: 1559-74.

Quirk FH, Heiman J, Rosen RC, et al (2002) Development of a sexual function questionnaire for clinical trials of female sexual function. Journal of Women's Health and Gender Based Medicine 11: 277-85.

Robins LN, Helzer JE, Weissman MM, et al (1984) Lifetime prevalence of specific psychiatric disorders in three sites. Archives of General Psychiatry 41: 949-58.

Rosen RC, Riley A, Wagner G, et al (1997) The International Index of Erectile Function (IIEF): a multi-dimensional scale for assessment of sexual dysfunction. Urology 49: 822-30.

Rosen RC, Brown C, Heiman J, et al (2000) The Female Sexual Function Index (FSFI): a multi-dimensional self-report instrument for the assessment of female sexual function. Journal of Sex and Marital Therapy 26: 191-208

Rosser BR, Metz ME, Bockting WO, et al (1997) Sexual difficulties, concerns, and satisfaction in homosexual men: an empirical study with implications for HIV prevention. Journal of Sex and Marital Therapy 23: $61-73$.

Rust J, Bennum I, Crowe M, et al (1989) The GRIMS: a psychometric instrument for the assessment of marital discord. In Sexuality Resulted Measures (eds CM Davis, WL Yarber, SL Davis): 223-5. Graphic Publishing.

Sebit MD (1995) Sexual dysfunction in African males in Nairobi, Kenya. East African Medical Journal 72: 631-4.

World Health Organization (1992) The ICD-10 Classification of Mental and Behavioural Disorders: Clinical Descriptions and Diagnostic Guidelines. World Health Organization.

${ }^{*}$ Recommended further reading
MCQs
d drug misuse
Select the single best option for each question stem
e alcohol misuse.
1 Classification of sexual dysfunction always enables us to:
a work with the couple
b engage with the couple
c commission services
d determine aetiology
e determine long-term prognosis.
3 In the assessment of sexual dysfunction:
$a$ it is essential to see the couple together
b determine the patient's sexual orientation
c always check blood pressure
d avoid discussion of sexual fantasy
e ignore past child abuse.

2 Sexual dysfunction is always associated
with:
a psychiatric disorder
b physical disorder
c gender identity
c between 25 and 35 years of age $d$ between 35 and 45 years of age e with anxiety.
5 Assessment of sexual dysfunction must always include:
a measurement of serum prolactin levels
b measurement of serum testosterone levels
c a thyroid function test
d liver function tests
e discussion of drug use. 